\title{
Wie moderne Technologie die Diabetes-Behandlung verbessert
}

\section{Roger Lehmann}

Prof. Dr. med., Leiter Diabetologie, Klinik für Endokrinologie, Diabetes und klinische Ernährung, UniversitätsSpital Zürich

Die neusten Entwicklungen in der Behandlung des Diabetes Typ 1 im Bereich von Glukosesensoren und Insulinpumpen werden kurzfristig zu einer Verteuerung der Therapie führen. Im Gegenzug wird sich jedoch auch die Lebensqualität der Patienten deutlich erhöhen und die langfristig gute Einstellung des Blutzuckers und das Vermeiden von Folgekomplikationen wird zu wesentlichen Einsparungen im Gesundheitswesen führen.

Etwa 25000 Personen in der Schweiz weisen einen Diabetes Typ 1 auf, bei dem die Insulin produzierenden Zellen der Bauchspeicheldrüse zerstört werden. Durch den Insulinmangel gelangt die Glukose aus dem Blut nicht in die Körperzellen, der Blutzuckerspiegel steigt. Dies verursacht nicht nur die Diabetes-typischen Krankheitssymptome, auch schwere Folgeerkrankungen wie Herz-Kreislauf-Erkrankungen, Sehverlust bis hin zur Erblindung, Nierenversagen oder Nervenschädigungen werden begünstigt.

Für die betroffenen Patienten bleibt es eine ständige Herausforderung, das fehlende Insulin zuzuführen und den Blutzuckerspiegel gut zu regulieren. In Kenntnis ihres aktuellen Zuckerwerts und abhängig von ihrer Nahrungsaufnahme und Aktivität müssen sie ihre Insulindosis anpassen und lebensgefährliche Unterzuckerungen oder Insulinmangel vermeiden.

\section{Fortschritte der Diabetestherapie im Leben eines 80-jährigen Patienten}

Wie sehr sich die Behandelbarkeit und Handhabbarkeit dieser Erkrankung verbessert hat, lässt sich gut an einem rüstigen 80-jährigen Patienten aufzeigen, der seit seinem 7. Lebensmonat einen Typ 1 Diabetes mellitus hat. Als er im Jahr 1937, 16 Jahre nach der Entdeckung des Insulins und 14 Jahre nach der InsulinMarkteinführung geboren wurde, existierte nur kurzwirksames Insulin, welches drei- bis viermal pro Tag mit einer Glaskolbenspritze injiziert werden musste, deren Stahl-Nadel in Alkohol desinfiziert und bei Bedarf mit einer Metallfeile nachgeschliffen wurde. Zur Zuckerbestimmung wurde der Urin mit dem BenedictTest auf einem Bunsenbrenner aufgekocht, erst 1941 brauchte es durch den Clinitest nur noch wenige Tropfen Urin und Wasser. Auf die Blutzuckermessung musste dieser Patient über 30 Jahre lang warten: Der Hämoglukosetest gewann in den 70er-Jahren langsam an Bedeutung. Die ersten digitalen Blutzuckergeräte kamen 1980 auf den Markt. Auch in der Insulinentwicklung hat dieser Patient vieles miterlebt: von der Einführung eines lange wirksamen Basalinsulins 1946 (Insulatard) bis hin zu den gentechnologisch hergestellten Human- und dann Analoginsulinen.

\section{Kontinuierliche Glukosemessung vermeidet Unterzuckerung}

Nach einer schweren Unterzuckerung, an der er fast gestorben wäre, wünscht er sich ein System zur kontinuierlichen Blutzuckermessung, das ihn vor Unterzuckerungen warnt. Kontinuierliche Glukosemesssysteme (CGM) sind seit 1999 in der Diabetes-Therapie im Einsatz. Dieser Patient entscheidet sich für ein CGMSystem, welches neben der Warnfunktion auch seine Kontaktpersonen via Smartphone alarmiert. Er erhält eine individuell angepasste Anwendungsschulung durch das Diabetesteam und lernt die Werte des Systems (absolute Glukosewerte und Trendinformationen) $\mathrm{zu}$ verstehen sowie geeignete Massnahmen zu ergreifen.

\section{Junge Frau mit einem neu entdeckten Typ-1-Diabetes mellitus}

Während eines Auslandaufenthalts wurde einer 21-jährigen Patientin ein Diabetes diagnostiziert und mit einem langwirksamen Insulin vor dem Schlafen und 
mit kurzwirksamen Insulin vor den Mahlzeiten therapiert (Basis-Bolus). Die Behandlung könnte im Vergleich zu 1937 unterschiedlicher nicht sein. Das Erlernen der Basis-Elemente wie Injektionstechnik, Blutzuckermessung und v.a. Erkennen und Schätzen von Kohlenhydraten blieb zwar gleich, aber die heutige Verwendung von ultra-kurzwirksamem Analoginsulin und ultra-lang wirksamem Basisinsulin erlaubt ein viel besseres Insulin- und Blutzuckerprofil. Seit Juli 2017 wird zudem bei allen Patienten mit einer solch intensivierten Insulintherapie mit der Flash-Glukose Messmethode (FGM) ein neues System für die Blutzuckermessung von den Krankenkassen übernommen. Die Patientin erhält gleich in der ersten Sprechstunde dieses System mit einem kleinen Stich und einem Kleber auf der Haut und nach einer Stunde kann sie den Zuckerwert im Gewebe über eine Zeitdauer von 14 Tagen beliebig oft messen. So kann sie nach jeder Mahlzeit einfach erkennen, ob die Balance zwischen Insulin und Kohlenhydraten gestimmt hat, oder ob etwas geändert werden muss. Sie ist auch befreit davon, sich 4-6 Mal pro Tag in den Finger stechen zu müssen, um den Blutzucker zu messen. Die meisten Patienten messen mit diesem neuen System den Zucker etwa 10-16 Mal pro Tag und die Grafik zeigt den Verlauf der letzten 8 Stunden an.

\section{Auf dem Weg zu einer «künstlichen Bauchspeicheldrüse»}

Nicht zuletzt auch weil diese einfachen FGM Systeme nicht vor einer Unterzuckerung warnen, was v.a. in der Nacht wichtig wäre, stellt sich bei dieser jungen Patientin früher oder später die Frage, ob nicht die Kombination eines Glukosesensors mit einer Insulinpumpe die Blutzuckereinstellung noch zusätzlich verbessern würde. Bei einer solchen sensorunterstützten Insulinpumpe kommuniziert der Sensor direkt mit der Pumpe, wobei letztere die Insulinzufuhr bereits vor dem Auftreten einer Unterzuckerung unterbrechen kann. Steigt der Glukosespiegel wieder an, wird die Insulinabgabe wieder aufgenommen. Die neueste Version dieser sensorunterstützten Insulinpumpe (670 G), welche bei uns voraussichtlich nächstes Jahr eingeführt wird, verhindert nicht nur Unterzuckerungen, sondern korrigiert auch hohe Blutzuckerwerte automatisch. Sie funktioniert damit wie eine halbautomatische künstliche Bauchspeicheldrüse. Halbautomatisch deshalb, weil immer noch die Insulinmenge für eine bestimmte Mahlzeit bestimmt und die Kohlenhydratmenge korrekt geschätzt werden muss. Nach Eingabe von Blutzucker und Kohlenhydratmenge wird die benötigte Insulinmenge aufgrund von individuellen Regeln berechnet. Eines der technologischen Ziele in der Diabetestherapie - die Entwicklung einer künstlichen Bauchspeicheldrüse, die Insulinabgabe und Glukosekontrolle automatisch und selbständig steuern kann - scheint in Anbetracht dieser Fortschritte nicht mehr allzu weit entfernt zu sein.

\section{Ersparnisse trotz initial höherer Kosten zu erwarten}

Der Gebrauch von digitalen Hilfsmitteln verbessert nicht nur die Lebensqualität dieser chronisch kranken Menschen und vereinfacht ihre Blutzuckereinstellung,

Das Vermeiden von Unterzuckerungen und von hohen Blutzuckerwerten führt zu einer wesentlichen Reduktion von Hospitalisationen und Komplikationen.

er verteuert auch die aktuelle Therapie. Darum werden CGM-Systeme nur unter der Voraussetzung vergütet, dass eine Kontrolle nach 6 Monaten eine Verbesserung zeigt. Da diese Systeme relativ neu sind, gibt es noch keine grossen prospektiven Studien zu ihrer Wirtschaftlichkeit, dennoch weiss man, dass das Vermeiden von Unterzuckerungen und von hohen Blutzuckerwerten zu einer wesentlichen Reduktion von Hospitalisationen und Komplikationen führt. Die Ersparnisse dürften die initial höheren Kosten kompensieren, ganz abgesehen davon, dass die Lebensqualität der Betroffenen und ihre Produktivität im Berufsleben deutlich gesteigert werden dürfte.

\section{Zusammenfassung}

Die Behandlungsoptionen für Patienten mit Typ-1-Diabetes haben sich seit der Entdeckung des Insulins, aber auch in der jüngsten Vergangenheit deutlich verbessert. Neue Systeme der Blutzuckermessung und Insulinabgabe erlauben ein kaum belastendes, ständiges Monitoring des Zuckerwerts und eine optimal abgestimmte Insulingabe. Dies wirkt sich nicht nur auf Alltag und Lebensqualität der Patienten positiv aus. Auch beeinträchtigende und kostspielige Folgeerkrankungen, Notfälle und Komplikationen werden verhindert. 